\title{
Single top quark production cross section at hadron colliders
}

\section{Nikolaos Kidonakis ${ }^{* \dagger}$}

Kennesaw State University, USA

E-mail: nkidonak@kennesaw.edu

\begin{abstract}
I present results for the theoretical cross section of single top quark production in the $s$ channel and also via associated production with a $\mathrm{W}$ boson or a charged Higgs. The calculations include higher-order corrections at NNLL accuracy. Detailed numerical results are provided for Tevatron and LHC energies.
\end{abstract}

XVIII International Workshop on Deep-Inelastic Scattering and Related Subjects April 19 -23, 2010

Convitto della Calza, Firenze, Italy

\footnotetext{
* Speaker.

${ }^{\dagger}$ This work was supported by the National Science Foundation under Grant No. PHY 0855421.
} 

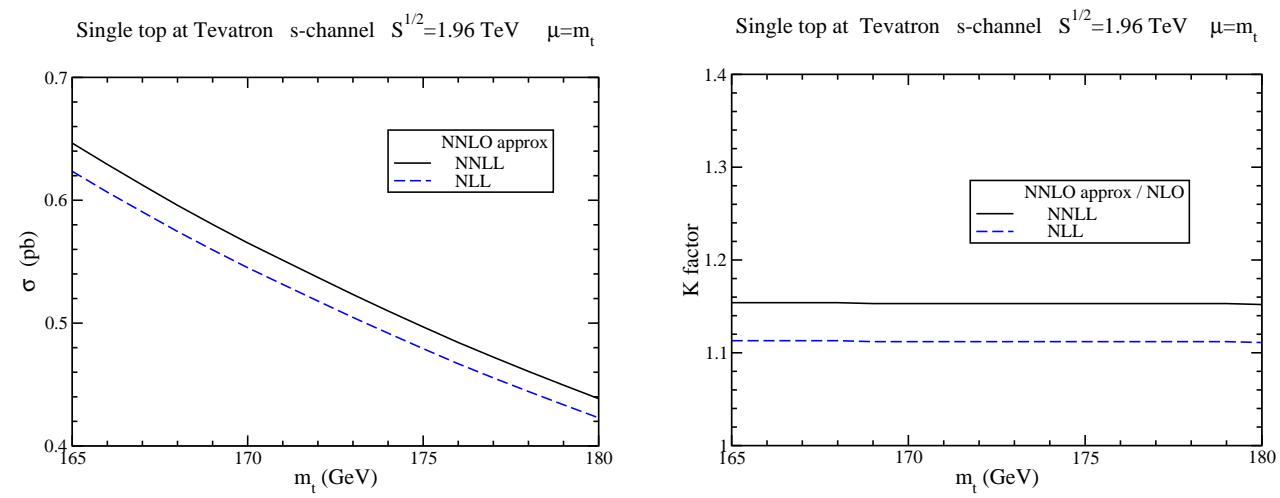

Figure 1: Cross section (left) and $K$ factor (right) for $s$-channel single top quark production at the Tevatron.

\section{Introduction}

Single top quark production has been obeserved at the Tevatron $[1,2]$ with a cross section consistent with Standard Model predictions. There are three partonic channels: the $t$-channel, the $s$-channel, and associated $t W^{-}$production. A related process to $t W^{-}$production is associated production of a top quark with a charged Higgs boson, $b g \rightarrow t H^{-}$.

The NLO corrections for the $s$ and $t$ channels were given in [3], for $t W^{-}$production in [4], and for $t H^{-}$production in [5, 6]. Higher-order QCD corrections are significant for single top quark production. Soft-gluon corrections resummed at next-to-leading-logarithm (NLL) accuracy have been calculated for the three single top channels in $[7,8]$ and for $t H^{-}$in $[9,10]$.

Recent developments in two-loop calculations of soft anomalous dimensions [11, 12] have now made it possible to improve the resummation to next-to-next-to-leading-logarithm (NNLL) accuracy. In this contribution I discuss new approximate NNLO calculations, derived from the expansion [13] of the NNLL resummed cross section, for $s$-channel production at the Tevatron and the LHC and for associated production of a top with a $W^{-}$or with a charged Higgs [12].

\section{Single top quark production in the $s$ channel}

The $s$-channel processes in single top quark production are $q \bar{q}^{\prime} \rightarrow \bar{b} t$ (i.e. $u \bar{d} \rightarrow \bar{b} t$, etc). We calculate the cross section at Tevatron and LHC energies using the MSTW2008 NNLO pdf [14].

At NLO the soft gluon corrections dominate the cross section and provide an excellent approximation to the complete NLO corrections, especially when damping factors are used to limit contributions far from threshold [12] (as are also used for $t \bar{t}$ production [15]). Thus it can be reasonably expected that at NNLO the soft-gluon corrections are dominant and that the NNLO cross section derived from NNLL resummation is a good approximation to the complete result.

In Fig. 1 we plot the cross section and the $K$ factor for $s$-channel single top quark production at the Tevatron. Results are shown for the NNLO approximate cross section from both NLL and NNLL resummation. The improved NNLL result increases the cross section even more than NLL, and provides a $15 \%$ enhancement over the NLO cross section. For a top quark mass of $173 \mathrm{GeV}$ 

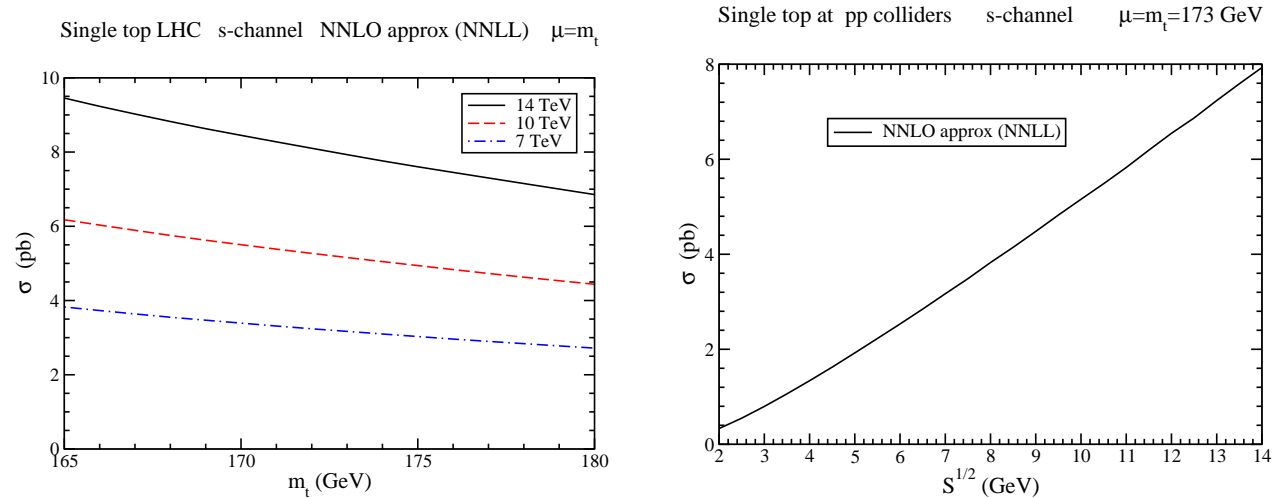

Figure 2: Cross section for $s$-channel single top quark production at the LHC versus mass (left) and energy (right).

Single antitop LHC s-channel NNLO approx (NNLL) $\mu=m_{t}$

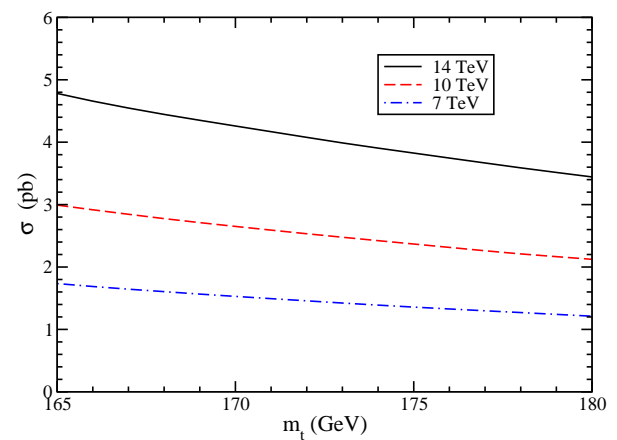

Single antitop at $\mathrm{pp}$ colliders s-channel $\quad \mu=\mathrm{m}_{\mathrm{t}}=173 \mathrm{GeV}$

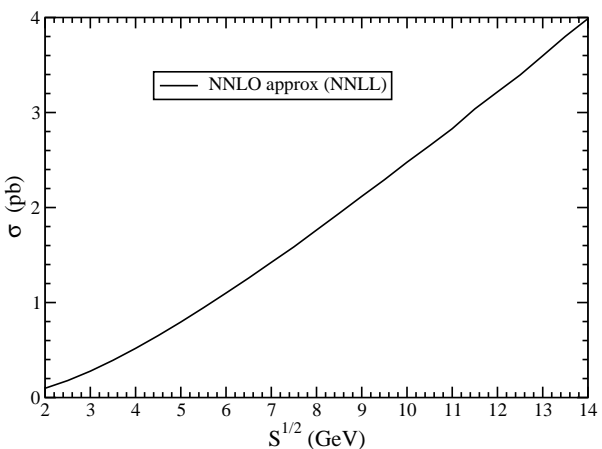

Figure 3: Cross section for $s$-channel single antitop production at the LHC vs mass (left) and energy (right).

we find

$$
\sigma_{s-\text { channel }}^{\text {NNLOapprox,top }}\left(m_{t}=173 \mathrm{GeV}, 1.96 \mathrm{TeV}\right)=0.523_{-0.005-0.028}^{+0.001+0.030} \mathrm{pb}
$$

where the first uncertainty is from scale variation and the second from pdf errors at $90 \%$ C.L. We note that the cross section for $s$-channel single antitop production at the Tevatron is identical to that for single top.

In Fig. 2 we plot the cross section for $s$-channel single top quark production at LHC energies. The left plot shows the NNLO approximate cross section from NNLL resummation versus the top quark mass for the current LHC energy of $7 \mathrm{TeV}$ as well as for energies of $10 \mathrm{TeV}$ and $14 \mathrm{TeV}$. The enhancement over NLO is around $13 \%$ at all three energies. The plot on the right shows the cross section as a function of c.m. energy at the LHC. For a top quark mass of $173 \mathrm{GeV}$ we find at $7 \mathrm{TeV}$

$$
\sigma_{s-\text { channel }}^{\text {NNLOapprox,top }}\left(m_{t}=173 \mathrm{GeV}, 7 \mathrm{TeV}\right)=3.17 \pm 0.06_{-0.10}^{+0.13} \mathrm{pb}
$$

while at $10 \mathrm{TeV}$ we have $5.16 \pm 0.09_{-0.14}^{+0.20} \mathrm{pb}$, and at $14 \mathrm{TeV}$ we have $7.93 \pm 0.14_{-0.28}^{+0.31} \mathrm{pb}$. 

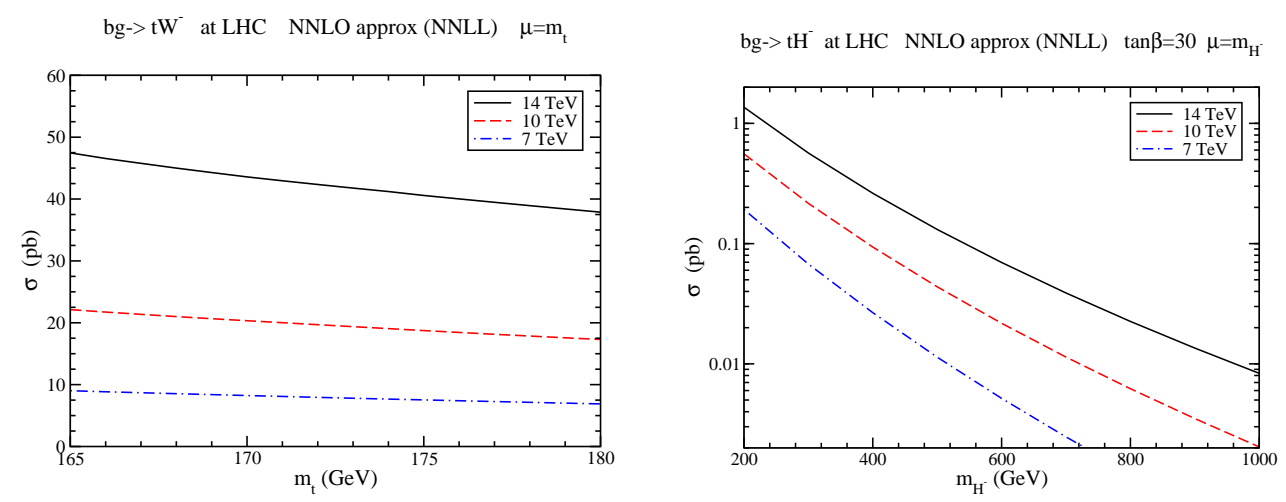

Figure 4: Cross section for $t W^{-}$(left) and $t H^{-}$(right) production at the LHC.

At the LHC the antitop s-channel cross section is different than for top. Figure 3 shows the corresponding results. At $7 \mathrm{TeV}$ we find

$$
\sigma_{s-\text { channel }}^{\text {NNLOaprox, antitop }}\left(m_{t}=173 \mathrm{GeV}, 7 \mathrm{TeV}\right)=1.42 \pm 0.01_{-0.07}^{+0.06} \mathrm{pb}
$$

while at $10 \mathrm{TeV}$ we have $2.48 \pm 0.02_{-0.13}^{+0.09} \mathrm{pb}$, and at $14 \mathrm{TeV}$ we have $3.99 \pm 0.05_{-0.21}^{+0.14} \mathrm{pb}$.

\section{Associated production of a top quark with a $W^{-}$}

We now turn to the process $b g \rightarrow t W^{-}$. This process is negligible at the Tevatron but significant at the LHC. The left plot of Fig. 4 shows the approximate NNLO cross section from NNLL resummation for $t W^{-}$production at LHC energies. The enhancement over NLO is around $8 \%$. At $7 \mathrm{TeV}$ with $m_{t}=173 \mathrm{GeV}$ we find

$$
\sigma_{t W^{-}}^{\text {NNLOapprox }}\left(m_{t}=173 \mathrm{GeV}, 7 \mathrm{TeV}\right)=7.8 \pm 0.2_{-0.6}^{+0.5} \mathrm{pb}
$$

while at $10 \mathrm{TeV}$ we have $19.4 \pm 0.5_{-1.1}^{+1.0} \mathrm{pb}$, and at $14 \mathrm{TeV}$ we have $41.8 \pm 1.0_{-2.4}^{+1.5} \mathrm{pb}$. Again, the first uncertainty is from scale variation and the second is from the MSTW2008 NNLO pdf at $90 \%$ C.L.

The cross section for $\bar{t} W^{+}$production is identical to that for $t W^{-}$.

\section{Associated production of a top quark with a charged Higgs}

Finally we consider the process $b g \rightarrow t H^{-}$. Charged Higgs bosons appear in supersymmetric theories, and the associated production of a top quark with an $H^{-}$is a potentially relevant process. The right plot of Fig. 4 shows the approximate NNLO cross section from NNLL resummation for $t H^{-}$production at LHC energies, for a value of $\tan \beta=30$, versus the charged Higgs mass. The NNLO approximate corrections increase the NLO cross section by $\sim 15 \%$ to $\sim 20 \%$ for the range of charged Higgs masses shown. 


\section{References}

[1] D0 Collaboration, V.M. Abazov et al., Observation of Single Top-Quark Production, Phys. Rev. Lett. 103, 092001 (2009), arXiv:0903.0850 [hep-ex]; Measurement of the t-channel single top quark production cross section, Phys. Lett. B 682, 363 (2010), arXiv:0907.4259 [hep-ex].

[2] CDF Collaboration, T. Aaltonen et al., Observation of Electroweak Single Top-Quark Production, Phys. Rev. Lett. 103, 092002 (2009), arXiv:0903.0885 [hep-ex].

[3] B.W. Harris, E. Laenen, L. Phaf, Z. Sullivan, and S. Weinzierl, Fully differential single-top-quark cross section in next-to-leading order QCD, Phys. Rev. D 66, 054024 (2002) [hep-ph/0207055].

[4] S.H. Zhu, Next-to-leading order QCD corrections to $b g \rightarrow t W^{-}$at CERN Large Hadron Collider, Phys. Lett. B 524, 283 (2002) [hep-ph/0109269]; (E) B 537, 351 (2002).

[5] S.H. Zhu, Complete next-to-leading order QCD corrections to charged Higgs boson associated production with a top quark at the CERN Large Hadron Collider, Phys. Rev. D 67, 075006 (2003) [hep-ph/0112109].

[6] T. Plehn, Charged Higgs boson production in bottom-gluon fusion, Phys. Rev. D 67, 014018 (2003) [hep-ph/0206121].

[7] N. Kidonakis, Single top quark production at the Fermilab Tevatron: Threshold resummation and finite-order soft gluon corrections, Phys. Rev. D 74, 114012 (2006) [hep-ph/0609287].

[8] N. Kidonakis, Higher-order soft gluon corrections in single top quark production at the CERN LHC, Phys. Rev. D 75, 071501(R) (2007) [hep-ph/0701080]; Soft and Collinear Enhancements to Top Quark and Higgs Cross Sections, Acta Phys. Polon. B 39, 1593 (2008), arXiv:0802.3381 [hep-ph]; Heavy-Flavor Production at Accelerators, Nucl. Phys. A 827, 448c (2009), arXiv:0901.2155 [hep-ph].

[9] N. Kidonakis, Charged Higgs production via $b g \rightarrow t H^{-}$at the LHC, JHEP 05 (2005) 011 [hep-ph/0412422].

[10] N. Kidonakis, Next-to-next-to-leading order soft and virtual corrections for QCD, Higgs and SUSY processes, Mod. Phys. Lett. A 19, 405 (2004) [hep-ph/0401147]; Higher order corrections to $H^{ \pm}$ production, POS (CHARGED2008) 003, arXiv:0811.4757 [hep-ph].

[11] N. Kidonakis, Two-Loop Soft Anomalous Dimensions and Next-to-Next-to-Leading-Logarithm Resummation for Heavy Quark Production, Phys. Rev. Lett. 102, 232003 (2009), arXiv:0903.2561 [hep-ph]; Two-loop soft anomalous dimensions with massive and massless quarks, in proceedings of DPF 2009, eConf C090726, arXiv:0910.0473 [hep-ph].

[12] N. Kidonakis, Next-to-next-to-leading logarithm resummation for s-channel single top quark production, Phys. Rev. D 81, 054028 (2010), arXiv:1001.5034 [hep-ph]; in these proceedings and in preparation.

[13] N. Kidonakis, Next-to-next-to-next-to-leading-order soft-gluon corrections in hard-scattering processes near threshold, Phys. Rev. D 73, 034001 (2006) [hep-ph/0509079].

[14] A.D. Martin, W.J. Stirling, R.S. Thorne, and G. Watt, Parton distributions for the LHC, Eur. Phys. J. C 63, 189 (2009), arXiv:0901.0002 [hep-ph].

[15] N. Kidonakis and R. Vogt, Next-to-next-to-leading order soft-gluon corrections in top quark hadroproduction, Phys. Rev. D 68, 114014 (2003) [hep-ph/0308222]; Theoretical top quark cross section at the Fermilab Tevatron and the CERN LHC, Phys. Rev. D 78, 074005 (2008), arXiv:0805.3844 [hep-ph]. 